\title{
Dual Cones and the Voronoi Algorithm
}

\author{
Jürgen Opgenorth
}

\section{CONTENTS}

Introduction

1. Dual Cones and Perfect Points

2. Discontinuous Groups

3. Bravais Manifolds of Finite Unimodular Groups

Acknowledgements

References
Max Koecher's axiomatic treatment of self-dual cones is generalized to pairs of dual cones. This leads to a powerful algorithm, à la Voronoi, to calculate the normalizer $N_{G L_{n}(\mathbb{Z})}(G)$ and to decide conjugacy in $G L_{n}(\mathbb{Z})$ for finite unimodular groups $G \leq G L_{n}(\mathbb{Z})$.

\section{INTRODUCTION}

Almost a century ago Voronoi [1908] formulated his fundamental algorithm to find the perfect (real positive definite quadratic) forms in $n$ variables. Among these one finds the forms representing the locally extreme lattice packings of spheres. The subject was taken up by M. Koecher [1960], who gave an axiomatic treatment of self-dual cones and a corresponding Voronoi algorithm in this situation aiming at the application of finding generators for certain arithmetic groups. Independently, the subject of extreme forms was taken up in [Bergé et al. 1992] to adjust Voronoi's algorithm to find the $G$-perfect forms, where $G$ is a finite unimodular group and the forms under consideration are $G$-invariant.

The key observation of the present paper is that a wide range of applications can be made if one generalizes Koecher's axioms to a pair of dual cones. The theory has here its natural setting and becomes more transparent; see Section 2. In particular, the perfect points live in one cone and the associated tessellation, which leads to Voronoi's neighbouring graph in the classical situation, lives in the dual cone. The context of discontinuously acting groups is treated in Section 3, where the quotient of the resulting Voronoi graph modulo this group action leads to a generating set for the group considered. As an application, the last section gives an algorithm to calculate normalizers of finite subgroups of $\mathrm{GL}_{n}(\mathbb{Z})$.

It turns out that the natural setup for a finite unimodular group $G$ is not just to look at the cone 
of positive definite $G$-invariant quadratic forms, but at the cone of forms invariant under the transposed group $G^{\text {tr }}$ as dual cone at the same time. Beyond the results of [Bergé et al. 1992] one gets a quite powerful algorithm to find a finite generating set for the normalizer of $G$ in the full unimodular group $\mathrm{GL}_{n}(\mathbb{Z})$ and an algorithm to decide conjugacy of two finite unimodular groups in the full unimodular group. These two problems have been open for a long time in the sense that the available finiteness proofs were constructive in principle but did not result in effective implementations. The algorithms are more effective than the ones I suggested in [Opgenorth 1996]. Meanwhile they have been used extensively and form a central part of CARAT (see [Opgenorth et al. 1998; Plesken and Schulz 2000]), a package for handling crystallographic groups up to degree 6 . The real critical parameter is not the degree, but the dimension of the space of invariant forms. Therefore some groups of degree beyond 24 could be handled.

\section{DUAL CONES AND PERFECT POINTS}

Let $\mathcal{V}_{1}, \mathcal{V}_{2}$ be real vector spaces of the same dimension $n$ and $\sigma: \mathcal{V}_{1} \times \mathcal{V}_{2} \longrightarrow \mathbb{R}$ a mapping that is linear and nondegenerate in both components. Two sets $\mathcal{V}_{1}^{>0} \subset \mathcal{V}_{1}$ and $\mathcal{V}_{2}^{>0} \subset \mathcal{V}_{2}$ are called dual cones (with respect to $\sigma$ ) if they satisfy the following axioms:

(DC1) $\mathcal{V}_{i}^{>0}$ is open in $\mathcal{V}_{i}$ and nonempty for $i=1,2$. (DC2) For all $x \in \mathcal{V}_{1}^{>0}$ and $y \in \mathcal{V}_{2}^{>0}$ one has $\sigma(x, y)>$ 0 .

(DC3) For every $x \in \mathcal{V}_{1} \backslash \mathcal{V}_{1}^{>0}$ there exists $0 \neq y \in$ $\mathcal{V}_{2}^{\geq 0}$ with $\sigma(x, y) \leq 0$ and for every $y \in \mathcal{V}_{2} \backslash \mathcal{V}_{2}^{>0}$ there exists $0 \neq x \in \mathcal{V}_{1}^{\geq 0}$ with $\sigma(x, y) \leq 0$. Here $\mathcal{V}_{i}^{\geq 0}$ denotes the closure of $\mathcal{V}_{i}^{>0}$ in $\mathcal{V}_{i}$ for $i=1,2$.

Obviously, this definition in symmetric in $\mathcal{V}_{1}$ and $\mathcal{V}_{2}$. For the remainder of this section let $\mathcal{V}_{1}^{>0}$ and $\nu_{2}^{>0}$ be fixed dual cones in $\mathcal{V}_{1}$ and $\mathcal{V}_{2}$, respectively, with respect to $\sigma$. For $i=1,2$ the boundary of $\mathcal{V}_{i}^{\geq 0}$ is denoted by $\partial \mathcal{V}_{i}^{>0}$. Some elementary properties are collected in the following lemma.

Lemma 1.1 [Koecher 1957]. (i) Let $x, y \in \mathcal{V}_{1}^{>0}$ and $a, b$ be positive real numbers. Then $a x+b y$ is also in $v_{1}^{>0}$.

(ii) For $0 \neq x \in \mathcal{V}_{1}^{\geq 0}$ and $y \in \mathcal{V}_{2}^{>0}$ one has $\sigma(x, y)>$ 0 . (iii) For every $x \in \mathcal{V}_{1} \backslash \mathcal{V}_{1}^{\geq 0}$ there exists $y \in \mathcal{V}_{2}^{>0}$ with $\sigma(x, y)<0$.

(iv) For every $0 \neq x \in \partial \mathcal{V}_{1}^{>0}$ there exists $0 \neq y \in$ $\partial \mathcal{V}_{2}^{>0}$ with $\sigma(x, y)=0$.

(v) $x \in \mathcal{V}_{1}^{\geq 0}$ and $-x \in \mathcal{V}_{1}^{\geq 0}$ implies $x=0$.

(vi) Let $\Phi_{2}$ be a positive definite scalar product on $\mathcal{V}_{2}$ and define $|y|_{2}:=\sqrt{\Phi_{2}(y, y)}$. For every compact subset $A \subset \mathcal{V}_{1}^{>0}$ there exists a real number $\rho(A)>$ 0 with $\sigma(a, y) \geq \rho(A)|y|_{2}$ for every $a \in A$ and $y \in \mathcal{V}_{2}^{\geq 0}$.

Proof. (i) Suppose $a x+b y \notin \mathcal{V}_{1}^{>0}$. Then by (DC3) there exists $z \in \mathcal{V}_{2}^{\geq 0}$ with $0 \geq \sigma(a x+b y, z)=$ $a \sigma(a, z)+b \sigma(y, z)$, but this gives a contradiction, since $a, b, \sigma(x, z)$ and $\sigma(y, z)$ are positive real numbers.

(ii) Let $0 \neq x \in \mathcal{V}_{1}^{\geq 0}$. Since $\sigma$ is continuous, (DC2) implies $\sigma(x, y) \geq 0$ for every $y \in \mathcal{V}_{2}^{>0}$. Suppose there exists $y \in \mathcal{V}_{2}^{>0}$ with $\sigma(x, y)=0$. Then $x \notin \mathcal{V}_{1}^{>0}$. By (DC1) $\mathcal{V}_{2}^{>0}$ is open. So for every $z \in \mathcal{V}_{2}$ there exists $\lambda>0$ such that $y+\lambda z \in \mathcal{V}_{2}^{>0}$. Hence

$$
0 \leq \sigma(x, y+\lambda z)=\sigma(x, y)+\lambda \sigma(x, z)=\lambda \sigma(x, z) \text {. }
$$

This implies $\sigma(x, z) \geq 0$ for every $z \in \mathcal{V}_{2}$ which gives a contradiction to the assumption that $\sigma$ is nondegenerate.

(iii) Suppose there exists $x \in \mathcal{V}_{1} \backslash \mathcal{V}_{1}^{\geq 0}$ with $\sigma(x, y) \geq$ 0 for all $y \in \mathcal{V}_{2}^{>0}$. The set $\mathcal{V}_{1} \backslash \mathcal{V}_{1}^{\geq 0}$ is open, so for every $x^{\prime} \in \mathcal{V}_{1}^{>0}$ there exists a positive real number $\lambda$ with $x+\lambda x^{\prime} \notin \mathcal{V}_{1}^{\geq 0}$. But by (DC3) there exits $0 \neq y^{\prime} \in \mathcal{V}_{2}^{\geq 0}$ with $\sigma\left(x+\lambda x^{\prime}, y^{\prime}\right) \leq 0$, hence

$0 \geq \sigma\left(x+\lambda x^{\prime}, y^{\prime}\right)=\sigma\left(x, y^{\prime}\right)+\lambda \sigma\left(x^{\prime}, y^{\prime}\right) \geq \lambda \sigma\left(x^{\prime}, y^{\prime}\right)$.

But $\sigma\left(x, y^{\prime}\right) \geq 0$ by assumption, $\lambda>0$ by assumption and $\sigma\left(x^{\prime}, y^{\prime}\right)>0$ by (ii). So we have a contradiction.

(iv) This can be proved with the same arguments used in (ii) and (iii).

(v) By (ii) $\sigma(x, y) \geq 0$ and $\sigma(-x, y) \geq 0$ for all $y \in$ $\mathcal{V}_{2}^{>0}$. Therefore $\sigma(x, y)=0$ for all $y \in \mathcal{V}_{2}^{>0}$. Now the claim follows from the fact that $\mathcal{V}_{2}^{>0}$ is open in $\mathcal{V}_{2}$ and $\sigma$ is nondegenerate.

(vi) The equation is homogeneous in $y$ on both sides, so it suffices to prove the statement for all $a \in A$ and $y \in \mathcal{V}_{2}^{\geq 0}$ with $|y|_{2}=1$. These tuples $(a, y)$ form a compact subset in $\mathcal{V}_{1}^{>0} \times \mathcal{V}_{2}^{\geq 0}$. Now $\sigma(a, y)$ is continuous, so the minimum is attained. By (ii) the minimum is positive. 
Let $D$ be a fixed subset of $\mathcal{V}_{2}^{\geq 0} \backslash\{0\}$, that is discrete in $\mathcal{V}_{2}$ and $x \in \mathcal{V}_{1}^{>0}$. Now Lemma 1.1(vi) implies $\sigma(x, d) \geq \rho(x)|d|_{2}$ for every $d \in D$. Consequently, $\sigma(x, d)>0$ for every $d \in D$ and the number of $d \in D$ with $\sigma(x, d) \leq c$ for a given constant $c \in \mathbb{R}$ is finite. So the following definition makes sense.

Definition 1.2. Let $D \subset \mathcal{V}_{2}^{\geq 0} \backslash\{0\}$ be discrete in $\mathcal{V}_{2}$ and $x \in \mathcal{V}_{1}^{>0}$.

(i) $\mu_{D}(x):=\min \{\sigma(x, d): d \in D\}$ is called the $D$ minimum of $x$.

(ii) $M_{D}(x):=\left\{d \in D: \mu_{D}(x)=\sigma(x, d)\right\}$ is called the set of minimal D-vectors of $x$. Obviously $M_{D}(x)$ is a finite set and $M_{D}(x)=M_{D}(\lambda x)$ for all $\lambda>0$.

(iii) $V_{D}(x):=\left\{\sum_{d \in M_{D}(x)} a_{d} d: a_{d} \in \mathbb{R}^{\geq 0}\right\}$ is called the $D$-Voronoi domain of $x$.

(iv) A vector $x \in \mathcal{V}_{1}^{>0}$ is called $D$-perfect if its $D$ Voronoi domain has a nonempty interior. The set of $D$-perfect vectors with $D$-minimum 1 is denoted by $P_{D}$. Note that $x \in \mathcal{V}_{1}^{>0}$ is $D$-perfect if and only if $\operatorname{dim} \operatorname{span} M_{D}(x)=n$.

Geometrically, the perfect vectors can be interpreted as follows. Consider an affine hyperplane in $\mathcal{V}_{2}$ containing $n$ linearly independent points of $D$. This hyperplane divides $V_{2}$ into two open half-spaces one of them containing 0 . If this half-space contains no other point of $D$, the vector $x \in \mathcal{V}_{1}^{>0}$ with $\sigma(x, h)=1$ for all $h$ in this hyperplane is an element of $P_{D}$. This gives a bijection between $P_{D}$ and affine hyperplanes containing $n$ linearly independent elements of $D$ and no elements of $D$ in the half-space containing 0 .

Lemma 1.3. Let $\mathcal{V}_{1}^{>0}, \mathcal{V}_{2}^{>0}$ be dual cones with respect to $\sigma$ and $D \subset \mathcal{V}_{2}^{\geq 0} \backslash\{0\}$ be discrete in $\mathcal{V}_{2}$.

(i) Let $x \in \mathcal{V}_{1}^{>0}$. There exists a neighbourhood $U$ of $x$ with $U \subset \mathcal{V}_{1}^{>0}$ and $M_{D}(u) \subseteq M_{D}(x)$ for all $u \in U$.

(ii) The function $\mu_{D}$ is continuous.

Proof. Let $B=\overline{B_{r}(x)}$ be a compact ball with radius $r$ and center $x$ that is contained in $\mathcal{V}_{1}^{>0}$. By Lemma 1.1(vi) there exists a constant $\rho(B)>0$ such that $\sigma(b, d) \geq \rho(B)|d|_{2}$ for every $b \in B$ and $d \in D$. Let $\varepsilon>0$ be arbitrary. Since $D$ is discrete, the set

$M:=\left\{d \in D: \sigma(b, d) \leq \mu_{D}(x)+\varepsilon\right.$ for some $\left.b \in B\right\}$ is finite and $M_{D}(x) \subseteq M$. Let $M^{\prime}:=M \backslash M_{D}(x)$. Let $\kappa$ be a real number with

$$
0<\kappa<\min \left(\{\varepsilon\} \cup\left\{\sigma(x, d)-\mu_{D}(x): d \in M^{\prime}\right\}\right) .
$$

Now choose $0<\delta<r$ such that $|\sigma(y, d)|<\kappa / 2$ for every $d \in M$ and $y \in B_{\delta}(0)$. This implies $x+y \in B$ for $y \in B_{\delta}(0)$. Consequently, for every $y \in B_{\delta}(0)$,

$$
\begin{aligned}
\sigma(x+y, d)=\sigma(x, d)+\sigma(y, d) & \left\{\begin{array}{l}
<\mu_{D}(x)+\kappa / 2 \text { for all } d \in M_{D}(x), \\
>\mu_{D}(x)+\kappa / 2 \text { for all } d \in M^{\prime}, \\
>\mu_{D}(x)+\kappa / 2 \text { for all } d \in D \backslash M .
\end{array}\right.
\end{aligned}
$$

So $\sigma(x+y, d)>\mu_{D}(x)+\varepsilon>\mu_{D}(x)+\kappa / 2$ for all $d \in D \backslash M$ and $x+y \in B$. This implies $M_{D}(x+y) \subseteq$ $M_{D}(x)$ for every $y \in B_{\delta}(0)$. So (i) is proved.

Part (ii) follows from $\left|\mu_{D}(x)-\mu_{D}(x+y)\right| \leq \kappa / 2$ for all $y \in B_{\delta}(0)$ and the fact that $\kappa$ was chosen arbitrarily.

We do not want to consider arbitrary sets $D$. For example $D$ should contain at least a basis for $\mathcal{V}_{2}$, otherwise the set $P_{D}$ would be empty.

Definition 1.4. A set $D \subseteq \mathcal{V}_{2}^{\geq 0} \backslash\{0\}$ that is discrete in $\mathcal{V}_{2}$ is called admissible if for every sequence $\left(x_{i}\right)_{i \in \mathbb{N}} \subset$ $\mathcal{V}_{1}^{>0}$ that converges to a point $x \in \partial \mathcal{V}_{1}^{>0}$ the sequence $\left(\mu_{D}\left(x_{i}\right)\right)_{i \in \mathbb{N}}$ converges to 0 .

Lemma 1.5. Let $D \subseteq \mathcal{V}_{2}^{\geq 0} \backslash\{0\}$ be discrete in $\mathcal{V}_{2}$. Then $D$ is admissible if and only if for every $x \in$ $\partial \mathcal{V}_{1}^{>0}$ and $\varepsilon>0$ there exists $d \in D$ with $\sigma(x, d)<\varepsilon$.

Proof. ' $\Longrightarrow$ ' part: Let $x \in \partial \mathcal{V}_{1}^{>0}$ and $\left(x_{i}\right)_{i \in \mathbb{N}} \subset \mathcal{V}_{1}^{>0}$ with $x_{i} \rightarrow 0$. The sequence $\left(x+x_{i}\right)_{i \in \mathbb{N}}$ lies in $\mathcal{V}_{1}^{>0}$ and converges towards $x$ and for every $i$ there exists $d_{i}$ in $D$ with

$$
\begin{aligned}
\mu_{D}\left(x+x_{i}\right) & =\sigma\left(x+x_{i}, d_{i}\right)=\sigma\left(x, d_{i}\right)+\sigma\left(x_{i}, d_{i}\right) \\
& \geq \sigma\left(x, d_{i}\right) \geq 0 .
\end{aligned}
$$

Now the claim follows from $\mu_{D}\left(x+x_{i}\right) \rightarrow 0$.

' $\Leftarrow$ ' part: Let $\left(x_{i}\right)_{i \in \mathbb{N}}$ be a sequence in $\mathcal{V}_{1}^{>0}$ converging to $x \in \partial \mathcal{V}_{1}^{>0}$. Choose $\varepsilon>0$ and $d \in D$ with $\sigma(x, d)<\varepsilon$. There exists $i_{0} \in \mathbb{N}$ with

$$
\left|\sigma\left(x_{i}-x, d\right)\right|<\varepsilon \quad \text { for all } i \geq i_{0} .
$$

Thus $\mu_{D}\left(x_{i}\right) \leq \sigma\left(x_{i}, d\right)=\sigma(x, d)-\sigma\left(x_{i}-x, d\right)<2 \varepsilon$ for all $i \geq i_{0}$, so $\mu_{D}\left(x_{i}\right) \rightarrow 0$.

Lemma 1.6. Let $D \subset \mathcal{V}_{2}^{>0} \backslash\{0\}$ be discrete in $\mathcal{V}_{2}$ and admissible. Then $P_{D}$ is a discrete set. 
Proof. Let $\left(x_{i}\right)_{i \in \mathbb{N}} \subseteq P_{D}$ converging to $x \in \mathcal{V}_{1}^{\geq 0}$. If $x$ is a boundary point of $\mathcal{V}_{1}^{>0}$ then $\mu_{D}\left(x_{i}\right) \rightarrow 0$, but this gives a contradiction since $\mu_{D}\left(x_{i}\right)=1$ for all $i \in \mathbb{N}$ by the definition of $P_{D}$.

Assume $x \in \mathcal{V}_{1}^{>0}$. By Lemma 1.3 there exists a neighbourhood $U$ of $x$ with $M_{D}(u) \subseteq M_{D}(x)$ for all $u \in U$. So there exists $i_{0} \in \mathbb{N}$ such that $M_{D}\left(x_{i}\right) \subseteq$ $M_{D}(x)$ for all $i \geq i_{0}$. Let $i \geq i_{0}$. Since $x_{i}$ is perfect there are linearly independent vectors $d_{1}, \ldots, d_{n} \in$ $M_{D}\left(x_{i}\right) \subseteq M_{D}(x)$. For these vectors one has

$$
\sigma\left(x_{i}-x / \mu_{D}(x), d_{j}\right)=0,
$$

for $1 \leq j \leq n$. Now $\sigma$ is nondegenerate. This implies that $x_{i}-x / \mu_{D}(x)=0$ for all $i \geq i_{0}$.

From the proof of this lemma there follows also a corollary:

Corollary 1.7. Let $x, y$ be D-perfect vectors and let $d_{1}, \ldots, d_{k}$, with $k \geq n$, be vectors from the intersection of $M_{D}(x)$ and $M_{D}(y)$ containing a basis of $\mathcal{V}_{2}$. Then there exists $0<\lambda \in \mathbb{R}$ such that $x=\lambda y$.

The proof of the following proposition shows a constructive method to calculate a $D$-perfect vector.

Proposition 1.8. If $D \subset \mathcal{V}_{2}^{\geq 0} \backslash\{0\}$ is discrete in $\mathcal{V}_{2}$ and admissible then for every $y \in \mathcal{V}_{1}^{>0}$ there exists $x \in P_{D}$ with $M_{D}(y) \subseteq M_{D}(x)$.

Proof. Let $k$ be the dimension of the span of $M_{D}(y)$. If $k=n$ we are done. So assume $k<n$. One can calculate $0 \neq z \in \mathcal{V}_{1}$ with $\sigma(z, d)=0$ for all $d \in M_{D}(y)$. Without loss of generality, $z \notin \mathcal{V}_{1}^{\geq 0}$ (otherwise take $-z$ ). Now consider $y+\lambda z$ for nonnegative real $\lambda$. By the choice of $z$ there exists a $\lambda_{0}$ such that $y+\lambda_{0} z \in \partial \mathcal{V}_{1}^{>0}$. By assumption $D$ is admissible, so $\mu_{D}(y+\lambda z) \rightarrow 0$ for $\lambda \rightarrow \lambda_{0}$. The continuity of $\mu_{D}$ implies that there is a $\lambda_{1} \in\left(0, \lambda_{0}\right)$ with $\mu_{D}(y)>\mu_{D}\left(y+\lambda_{1} z\right)>0$. Let $M$ be the finite set of all $d \in D$ with $\sigma\left(y+\lambda_{1} z, d\right) \leq \mu_{D}(y)$. Clearly $M_{D}(y)$ is a proper subset of $M$, and for all $d \in M^{\prime}:=M \backslash M_{D}(y)$ one has $\sigma(z, d)<0$ and $\sigma(y, d)>\mu_{D}(y)$. Define

$$
\begin{aligned}
\lambda_{2} & :=\min \left\{\frac{\mu_{D}(y)-\sigma(y, d)}{\sigma(z, d)}: d \in M^{\prime}\right\}, \\
y_{2} & :=y+\lambda_{2} z .
\end{aligned}
$$

Obviously $0<\lambda_{2}<\lambda_{1}$ and for all $d \in D \backslash M$ one has $\sigma\left(y_{2}, d\right)>\mu_{D}(y)$ since $\sigma(y, d)>\mu_{D}(y)$ and $\sigma\left(y+\lambda_{1} z, d\right)>\mu_{D}(y)$. By construction $\sigma\left(y_{2}, d\right) \geq$
$\mu_{D}(y)$ for all $d \in M$ and equality is obtained for all $d \in M_{D}(y)$ and at least one $d \in M^{\prime}$. This $d$ cannot be in the span of $M_{D}(y)$ since $z \in M_{D}(y)^{\perp}$ and hence otherwise $\mu_{D}(y)=\sigma\left(y_{2}, d\right)=\sigma(y, d)$, which implies $d \in M_{D}(y)$. So $M_{D}(y) \subset M_{D}\left(y_{2}\right)$ and $\operatorname{dim}\left\langle M_{D}\left(y_{2}\right)\right\rangle>k$. Iteration of this procedure yields the desired result.

Next we want to consider the $D$-Voronoi domain of a $D$-perfect vector $x$. It is defined as the set of linear combinations with nonnegative coefficients of the finitely many vectors in $M_{D}(x)$ and forms a cone in $V_{2}^{\geq 0}$ based at the origin. This cone can be described in a dual way by finitely many linear inequalities of the form $\sigma(y,) \geq$.0 with certain $y \in \mathcal{V}_{1}$. A vector $0 \neq y \in \mathcal{V}_{1}$ with $\sigma(y, z) \geq 0$ for every $z \in M_{D}(x)$ and $\sigma(y, z)=0$ for $n-1$ linearly independent $z \in M_{D}(x)$ is called a direction for $x$. The directions of $x$ correspond with the walls of $V_{D}(x)$, i.e., for a direction $y$ of $x$ the set $W(y):=V_{D}(x) \cap\left\{z \in \mathcal{V}_{2}: \sigma(y, z)=0\right\}$ belongs to the boundary of $V_{D}(x)$ and is a cone of dimension $n-1$ in $\mathcal{V}_{2}$.

If a direction $y$ of $x$ lies in $V_{1}^{\geq 0}$, then

(i) $\sigma(y, d) \geq 0$ for all $d \in D$ and

(ii) $\sigma(y, z)=0$ for all $z \in W(y)$.

From (i) it follows that $M_{D}(x+\lambda y)=M_{D}(x) \cap$ $W(y)$, so for any $\lambda>0$ the vector $x+\lambda y$ is not $D$-perfect. The second point shows that $W(y)$ is in the boundary of $\mathcal{V}_{2}^{\geq 0}$ and also that $y$ is a boundary point of $\mathcal{V}_{1}^{\geq 0}$. Therefore the directions of $x$ that are in $\mathcal{V}_{1}^{\geq 0}$ are called blind directions. Otherwise, if $y$ is not contained in $\mathcal{V}_{2}^{\geq 0}$ there exists for admissible $D$ a vector $d \in D$ with $\sigma(y, d)<0$. As shown in the proof of 1.8 one can find a $\lambda>0$ such that $x+\lambda y$ is $D$-perfect and $M_{D}(x) \cap M_{D}(x+\lambda y)$ generates $W(y)$. The vector $x+\lambda y$ is called a neighbour of $x$ (in the direction $y$ ).

Theorem 1.9. If $D \subset \mathcal{V}_{2}^{\geq 0} \backslash\{0\}$ is discrete in $\mathcal{V}_{2}$ and admissible then the $D$-Voronoi domains of the $D$ perfect vectors form an exact tessellation of $\mathcal{V}_{2}^{>0}$. Exact means, that every wall of a D-Voronoi domain is a wall of exactly two D-Voronoi domains.

Proof. Let $x_{1}, x_{2} \in P_{D}$ with $x_{1} \neq x_{2}$. We have to show that $V_{D}\left(x_{1}\right)$ and $V_{D}\left(x_{2}\right)$ have no interior point in common. Suppose $x \in \operatorname{Int}\left(V_{D}\left(x_{1}\right)\right) \cap \operatorname{Int}\left(V_{D}\left(x_{2}\right)\right)$. Without loss of generality, assume $\sigma\left(x_{1}, x\right)=1$ and 
$\sigma\left(x_{2}, x\right) \leq 1$. There exist $d_{1}, \ldots, d_{k} \in M_{D}\left(x_{1}\right), k \geq$ $n$, containing a basis of $\mathcal{V}_{2}$, with

$$
x=\lambda_{1} d_{1}+\cdots+\lambda_{k} d_{k}
$$

for all $\lambda_{i}>0$ with $\sum_{i=1}^{k} \lambda_{i}=1$. By Corollary 1.7 at least one of the $d_{i}$, say $d_{1}$, is not in $M_{D}\left(x_{2}\right)$. Now

$$
\sigma\left(x_{2}, x\right)=\lambda_{1} \sigma\left(x_{2}, d_{1}\right)+\sum_{i=2}^{k} \lambda_{i} \sigma\left(x_{2}, d_{i}\right) .
$$

Each summand $\lambda_{i} \sigma\left(x, d_{i}\right)$ is greater or equal $\lambda_{i}$ and equality does not hold for the first one. Consequently $\sigma\left(x_{2}, x\right)>\sum_{i=1}^{k} \lambda_{i}=1$. But this is a contradiction to our assumption $\sigma\left(x_{2}, x\right) \leq 1$.

Exactness follows from the fact that for every neighbour $y$ of a $D$-perfect vector $x$ the $D$-Voronoi domain $V_{D}(x)$ and $V_{D}(y)$ have a common wall.

The next stage to show is that every $D$-Voronoi domain of $x \in P_{D}$ has only finitely many neighbours. We have shown the exactness, so it suffices to show that for every $d \in D \cap \mathcal{V}_{2}^{>0}$ the set $\left\{x \in P_{D}: d \in M_{D}(x)\right\}$ is finite, but this follows from Lemma 1.1(vi) and the fact that $P_{D}$ is discrete.

It remains to show that the $D$-Voronoi domains cover $\mathcal{V}_{2}^{>0}$. Let $y \in \mathcal{V}_{2}^{>0}$ and $x \in P_{D}$. If $y \notin V_{D}(x)$ then there exists a direction $z$ of $x$ with $\sigma(z, y)<0$. Let $x_{1}:=x+\lambda z$ be the neighbour of $x$ in the direction $z$. Since $\lambda>0$ one has $\sigma\left(x_{1}, y\right)<\sigma(x, y)$. This leads to a sequence $x, x_{1}, x_{2}, \ldots$ with $\sigma(x, y)>$ $\sigma\left(x_{1}, y\right)>\ldots$ Now Lemma 1.1(vi) and the discreteness of $P_{D}$ imply that there are only a finite number of $z \in P_{D}$ with $\sigma(z, y)<\sigma(x, y)$. Consequently there exists $k \in \mathbb{N}$ with $y \in V_{D}\left(x_{k}\right)$.

Definition 1.10. The graph $\Gamma_{D}$ of $D$-perfect vectors is the graph with vertex set $P_{D}$ and edge set $E=$ $\left\{(x, y) \in P_{D} \times P_{D}: x\right.$ and $y$ are neighbours $\}$.

The next corollary follows from Theorem 1.9.

Corollary 1.11. Let $D \subseteq \mathcal{V}_{2}^{\geq 0} \backslash\{0\}$ be discrete and admissible. Then $\Gamma_{D}$ is a connected, locally finite graph.

\section{DISCONTINUOUS GROUPS}

In this section we want to consider sets $D$ that are invariant under a group acting properly discontinuously on $\mathcal{V}_{2}^{>0}$. Let $\mathcal{V}_{1}^{>0}, \mathcal{V}_{2}^{>0}$ be dual cones with respect to $\sigma$. For $i=1,2$ we define

$$
\text { Aut } \mathcal{V}_{i}^{>0}:=\left\{g \in \operatorname{GL}\left(\mathcal{V}_{i}\right): \mathcal{V}_{i}^{>0} g=\mathcal{V}_{i}^{>0}\right\} \text {. }
$$

Let $\Omega$ be a subgroup of Aut $\mathcal{V}_{1}^{>0}$ that acts properly discontinuously on $\mathcal{V}_{1}^{>0}$, that is,

(i) for every $x \in \mathcal{V}_{1}^{>0}$ the stabilizer $\Omega_{x}:=\{w \in \Omega$ : $x w=x\}$ is finite, and

(ii) for every $x \in \mathcal{V}_{1}^{>0}$ the orbit $x \Omega:=\{x w: w \in \Omega\}$ has no cluster point in $\mathcal{V}_{1}^{\geq 0}$.

Then the group $\Omega^{\text {ad }}:=\left\{w^{\text {ad }}: w \in \Omega\right\}$ acts on $\mathcal{V}_{2}^{>0}$ where $w^{\text {ad }}$ is the unique element in $\operatorname{GL}\left(\mathcal{V}_{2}\right)$ with $\sigma(x w, y)=\sigma\left(x, y w^{\text {ad }}\right)$ for every $x \in \mathcal{V}_{1}$ and $y \in \mathcal{V}_{2}$.

For every $x \in \mathcal{V}_{1}^{>0}$ the set $H(x):=\left\{y \in \mathcal{V}_{2}^{\geq 0}\right.$ : $\sigma(x, y)=1\}$ is a compact, convex subset of $\mathcal{V}_{2}^{\geq 0}$. (That $H(x)$ is bounded follows from Lemma 1.1). If we denote by $x^{*}$ the center of mass of $H(x)$, then * defines a map from $\mathcal{V}_{1}^{>0}$ to $\mathcal{V}_{2}^{>0}$ with the property

$$
(x g)^{*}=x^{*} g^{- \text {ad }} \text { for all } x \in \mathcal{V}_{1}^{>0}, g \in \text { Aut } \mathcal{V}_{1}^{>0}
$$

because $g^{- \text {ad }}$ is a linear invertible map that maps $H(x)$ to $H(x g)$ and the center of mass is respected by linear mappings [Vinberg 1989, p. 28].

The action of $\Omega^{\text {ad }}$ on $\mathcal{V}_{2}^{>0}$ is again properly discontinuous. To prove this we assume that $\Omega^{\text {ad }}$ acts properly discontinuously and that $\Omega$ does not. (The roles of $\Omega$ and $\Omega^{\text {ad }}$ are swapped for simplicity of notation). If the stabilizer $\Omega_{x}$ of a point $x \in \mathcal{V}_{1}^{>0}$ is not finite, then $\left(\Omega_{x}\right)^{\text {ad }}$ fixes $x^{*}$ which contradicts the properly discontinuous action of $\Omega^{\text {ad }}$. If the orbit $x \Omega$ of $x \in \mathcal{V}_{1}^{>0}$ has a cluster point $x_{0}$ in $\mathcal{V}_{1}^{>0}$ then $x^{*} \Omega^{\text {ad }}$ has a cluster point $x_{0}^{*}$ because ${ }^{*}$ is obviously continuous.

Now we consider the case that $D$ is a subset of $\mathcal{V}_{2}^{\geq 0} \backslash\{0\}$, discrete in $\mathcal{V}_{2}^{>0}$, admissible and invariant under the action of $\Omega^{\text {ad }}$.

Lemma 2.1. Let $x \in \mathcal{V}_{1}^{>0}$ and $w \in \Omega$. Then

(i) $\mu_{D}(x w)=\mu_{D}(x)$,

(ii) $M_{D}(x w)=M_{D}(x)\left(\omega^{\text {ad }}\right)^{-1}$,

(iii) $V_{D}(x w)=V_{D}(x)\left(\omega^{\text {ad }}\right)^{-1}$.

In particular, $\Omega$ acts on the graph $\Gamma_{D}$.

Proof. Let $d \in M_{D}(x)$. Then

$$
\begin{aligned}
\mu_{D}(x) & =\sigma(x, d)=\sigma\left(x w w^{-1}, d\right) \\
& =\sigma\left(x w, d\left(w^{\mathrm{ad}}\right)^{-1}\right) \geq \mu_{D}(x w) .
\end{aligned}
$$

Applying $w^{-1}$ to $x w$ one gets $\mu_{D}(x w) \geq \mu_{D}(x)$. Using $d^{\prime} w^{-1}$ with $d^{\prime} \in M_{D}(x w)$ instead of $d \in M_{D}(x)$ in the above equation shows (ii) and (iii) follows from (ii). 
If $\Omega$ has only finitely many orbits on $\Gamma_{D}$ one can apply the theorem of Bass and Serre [Serre 1977; Dicks 1980, p. 21]. Together with the results in the previous section one gets the following theorem that allows the reconstruction of the group from the finite graph $\Gamma_{D} / \Omega$.

Theorem 2.2. Let $D \subset \mathcal{V}_{2}^{\geq 0} \backslash\{0\}$ be admissible and discrete in $\mathcal{V}_{2}$ and $\Omega \leq$ Aut $\mathcal{V}_{1}^{>0}$ acting properly discontinuously on $\mathcal{V}_{1}^{>0}$ such that $D$ is $\Omega^{\text {ad }}$-invariant and the residue graph $\Gamma_{D} / \Omega$ is finite. Let $x_{1}, \ldots, x_{l}$ be representatives of the $D$-perfect points spanning a connected subtree $T$ of $\Gamma_{D}$ and $T_{1}$ the finite set of all $y \in \Gamma_{D}-T$ that have a neighbour in $T$. For every $y \in T_{1}$ choose an element $w_{y} \in \Omega$ with $w_{y}^{-1}(y) \in$ $\left\{x_{1}, \ldots, x_{l}\right\}$. Then

$$
\Omega=\left\langle w_{y}, \operatorname{Stab}_{\Omega} x_{1}, \ldots, \operatorname{Stab}_{\Omega} x_{l}: y \in T_{1}\right\rangle,
$$

where $\operatorname{Stab}_{\Omega} x$ denotes the stabilizer of $x$ in $\Omega$. In particular the group $\Omega$ is finitely generated.

This theorem can be formulated as an algorithm in the following way.

1. Calculate a $D$-perfect vector $x_{1}$. Set $L_{1}=\left\{x_{1}\right\}$, $L_{2}=\varnothing$, and $S=\varnothing$.

2. If $L_{1}=\varnothing$ terminate, else take $x \in L_{1}$

3. Calculate a generating set $S_{x}$ for $\operatorname{Stab}_{\Omega} x$ and set $S=S \cup S_{x}$.

4. Calculate the set $R$ of directions of $x$ and a set $R^{\prime}$ of representatives of the orbits of $\operatorname{Stab}_{\Omega} x$ on $R$.

5. Calculate $N\left(R^{\prime}\right)=\left\{y \in P_{D}: y\right.$ is a neighbour of $x$ in direction $\left.r \in R^{\prime}\right\}$.

6. For every $y \in N\left(R^{\prime}\right)$ check if there exists a $z \in$ $L_{1} \cup L_{2}$ and a $w \in \Omega$ with $y w=z$.

If no such $z$ exists set $L_{1}=L_{1} \cup\{y\}$. If $z \in L_{1}$ set $S=S \cup\{w\}$.

7. Set $L_{2}=L_{2} \cup\{x\}, L_{1}=L_{1} \backslash\{x\}$. Go to step 2 .

Now $L_{2}$ is a set of representatives of $P_{D} / \Omega$, and $S$ is a generating set for $\Omega$.

Remark 2.3. If one wants to apply Theorem 2.2 to a special example of a group $\Omega$ acting properly discontinuously on a cone $\mathcal{V}_{1}^{>0}$, one has to consider the following problems.

(i) Find a dual cone $\mathcal{V}_{2}^{>0}$ and a bilinear form $\sigma$ such that $\mathcal{V}_{1}^{>0}$ and $\mathcal{V}_{2}^{>0}$ are dual cones with respect to $\sigma$. (ii) Find a subset $D \subset \mathcal{V}_{2}^{\geq 0} \backslash\{0\}$, discrete in $\mathcal{V}_{2}$, admissible and invariant under $\Omega^{\text {ad }}$.

(iii) For $x \in \mathcal{V}_{1}$ find a method to calculate $M_{D}(x)$.

(iv) Calculate the directions for $x$. There exists an algorithm to solve this [Opgenorth 1996].

(v) Prove that $\Omega$ has only finitely many orbits on $P_{D}$.

(vi) Find a method to calculate a generating set for the stabilizer in $\Omega$ for $D$-perfect vectors and to calculate an element $w \in \Omega$ with $x_{1} w=x_{2}$ for $x_{1}, x_{2}$ in $P_{D}$.

Remark 2.4. The stabilizer of a perfect vector $x$ permutes the directions of $x$. Since the directions of a $D$-perfect vector contain a basis $\left(b_{1}, \ldots, b_{n}\right)$ of $\mathcal{V}_{1}$ every $w \in \operatorname{Stab}_{\Omega} x$ is uniquely determined by the permutation of the directions. This gives a theoretical, but in general inefficient method of calculating the stabilizer. A similar argument holds for testing whether two $D$-perfect vectors are in the same orbit under $\Omega$ or not.

In the examples given in Section 3 we describe better ways of doing these calculations.

The last proposition of this section gives a sufficient criterion for the finiteness of $\Gamma_{D} / \Omega$ when $D$ is the intersection of the closed cone $\mathcal{V}_{2}^{\geq 0}$ with a lattice.

Proposition 2.5. Let $L$ be a lattice of full rank in $\mathcal{V}_{2}$, $D=L \cap \mathcal{V}_{2}^{\geq 0} \backslash\{0\}$ and $\Omega$ a subgroup of Aut $\mathcal{V}_{1}^{>0}$ that acts properly discontinuously on $\mathcal{V}_{1}^{>0}$. Assume that $D$ is admissible. If there exists a finite subset $V$ of $D$ such that the cone

$$
C=\left\{\sum_{v \in V} a_{v} v: a_{v} \in \mathbb{R}, a_{v} \geq 0\right\}
$$

contains a fundamental domain for the action of $\Omega^{\mathrm{ad}}$ on $\mathcal{V}_{2}^{>0}$, then $\Gamma_{D} / \Omega$ is a finite graph.

Proof. We define a subset

$$
M=\left\{y \in D: y_{V}-y \in \mathcal{V}_{2}^{\geq 0}\right\} \subset D,
$$

where $y_{V}:=n \sum_{v \in V} v$ and $n$ is the dimension of $\mathcal{V}_{2}$. For an arbitrary $x \in \mathcal{V}_{1}^{>0}$ and $y \in M$ one has $\sigma(x, y) \leq \sigma(x, y)+\sigma\left(x, y_{V}-y\right)=\sigma\left(x, y_{D}\right)$, this shows together with Lemma 1.1 the finiteness of $M$.

Let $x \in P_{D}$ and $d_{1}, \ldots, d_{n} \in M_{D}(x)$ linearly independent. The cone $C$ contains a fundamental domain for the action of $\Omega^{\text {ad }}$ on $\nu_{2}^{>0}$, so one can assume without loss of generality that the sum $d_{0}:=$ $d_{1}+\cdots+d_{n}$ is contained in $C$. This implies that $d_{0}$ 
can be written as $d_{0}=\sum_{v \in V} a_{v} v$ with nonnegative coefficients $a_{v}$. Now

$$
n=\sigma\left(x, d_{0}\right)=\sum_{v \in V} a_{v} \sigma(x, v) .
$$

But $\sigma(x, v) \geq 1$ for every $v \in V$, so $0 \leq a_{v} \leq n$ and $y_{V}-d_{0}=\sum_{v \in V}\left(n-a_{v}\right) v \in \mathcal{V}_{2}^{\geq 0}$. Also

$$
y_{V}-d_{i}=y_{V}-d_{0}+\sum_{j=1, j \neq i}^{n} d_{j} \in \mathcal{V}_{2}^{\geq 0}
$$

for $1 \leq i \leq n$; that is, $d_{i} \in M$. Thus we have proved that for every $x \in P_{D}$ there exists an element $w \in \Omega$ such that $M \cap M_{D}(x w)$ contains $n$ linear independent elements.

It was shown in the proof of Lemma 1.6 that a vector $x \in P_{D}$ is uniquely determined by any subset of $M_{D}(x)$ that contains a basis of $\nu_{2}$. Since $M$ is finite there are only finitely many subsets of $M$ that contain a basis of $\mathcal{V}_{2}$, and consequently there are only finitely many $x \in P_{D}$ such that $M \cap M_{D}(x)$ contains $n$ linearly independent vectors.

\section{BRAVAIS MANIFOLDS OF FINITE UNIMODULAR GROUPS}

In this section the methods developed in Section 2 are used to calculate the normalizer of a finite unimodular group. This is done by calculating the graph of $G$-perfect forms. In his classical paper Voronoi treated the case where $G$ is the trivial group represented by the $d \times d$ unit-matrix [Voronoi 1908]. In this case the normalizer is all of $\mathrm{GL}_{d}(\mathbb{Z})$ acting on the space of symmetric $d \times d$ matrices, denoted by $\mathbb{R}_{\mathrm{sym}}^{d \times d}$.

Definition 3.1. Let $G$ be a subgroup of $\mathrm{GL}_{d}(\mathbb{Z})$.

(i) The normalizer $N_{\mathbb{Z}}(G)$ of $G$ is defined by

$$
N_{\mathbb{Z}}(G):=\left\{h \in \mathrm{GL}_{d}(\mathbb{Z}): h^{-1} G h=G\right\} .
$$

(ii) $\mathcal{F}(G):=\left\{A \in \mathbb{R}_{\text {sym }}^{d \times d}: g A g^{\text {tr }}=A\right.$ for all $\left.g \in G\right\}$ is called the space of invariant forms of $G$. The set of the positive semidefinite elements in $\mathcal{F}(G)$ is denoted by $\mathcal{F}^{\geq 0}(G)$. The set of positive definite denoted is denoted by $\mathcal{F} \geq 0(G)$ and is called the Bravais manifold of $G$. $\left(\mathcal{F}^{>0}(G)\right.$ is nonempty if and only if $G$ is finite.)

(iii) For a subset $X$ of $\mathbb{R}_{\mathrm{sym}}^{d \times d}$ the Bravais group of $X$ is defined by $B(X):=\left\{g \in \mathrm{GL}_{d}(\mathbb{Z}): g A g^{\mathrm{tr}}=A\right.$ for all $\left.A \in X\right\}$. (iv) $\mathcal{B}(G):=\mathcal{B}(\mathcal{F}(G))$ is called the Bravais group of $G$. A group $G$ is called a Bravais group if $G=$ $\mathcal{B}(G)$.

Remark 3.2 (compare [Brown et al. 1973; Opgenorth 1996]). Let $G \leq \mathrm{GL}_{d}(\mathbb{Z})$ be finite.

(i) $\mathcal{B}(G)$ is finite and $\mathcal{B}(\mathcal{B}(G))=\mathcal{B}(G)$.

(ii) $\mathcal{F}^{>0}(G)$ is an open cone in $\mathcal{F}(G)$.

(iii) $N_{\mathbb{Z}}(G)$ acts properly discontinuously on $\mathcal{F}^{>0}(G)$, by $A \mapsto h^{-1} A h^{- \text {tr }}$ for $h \in N_{\mathbb{Z}}(G)$.

(iv) $N_{\mathbb{Z}}(\mathcal{B}(G))=\left\{h \in \mathrm{GL}_{d}(\mathbb{Z}): h^{-1} \mathcal{F}(G) h^{- \text {tr }}=\right.$ $\mathcal{F}(G)\}$.

(v) $N_{\mathbb{Z}}(G)$ is a subgroup of finite index in $N_{\mathbb{Z}}(\mathcal{B}(G)$ ).

Lemma 3.3. Let $G$ be a finite subgroup of $\mathrm{GL}_{d}(\mathbb{Z})$. Then $\mathcal{F}^{>0}(G)$ and $\mathcal{F}^{>0}\left(G^{\text {tr }}\right)$ are dual cones with respect to

$$
\sigma: \mathcal{F}(G) \times \mathcal{F}\left(G^{\mathrm{tr}}\right) \longrightarrow \mathbb{R}:(A, B) \mapsto \operatorname{trace}(A B) .
$$

Proof. Axiom (DC1) has already been stated in Remark 3.2(ii).

The positive definite matrices in $\mathbb{R}_{\mathrm{sym}}^{d \times d}$ form a selfdual cone with respect to $\sigma$ [Koecher 1960; Opgenorth 1996]; this implies (DC2).

To prove (DC3) let $A \in \mathcal{F}(G) \backslash \mathcal{F}>0(G)$ and $B \in$ $\mathbb{R}_{\text {sym }}^{d \times d}$ positive definite with $\operatorname{trace}(A B) \leq 0$. Then $C:=\sum_{g \in G} g^{\operatorname{tr}} B g$ is a positive definite matrix in $\mathcal{F}^{>0}\left(G^{\text {tr }}\right)$ with

$$
\begin{aligned}
\sigma(A, C) & =\sum_{g \in G} \operatorname{trace}\left(A g^{\operatorname{tr}} B g\right)=\sum_{g \in G} \operatorname{trace}\left(g A g^{\operatorname{tr}} B\right) \\
& =|G| \operatorname{trace}(A B) .
\end{aligned}
$$

Remark 3.4. It follows from the proof of Lemma 3.3 that the map

$$
\pi: \mathbb{R}_{\mathrm{sym}}^{d \times d} \longrightarrow \mathcal{F}\left(G^{\mathrm{tr}}\right): A \mapsto \frac{1}{|G|} \sum_{g \in G} g^{\mathrm{tr}} A g
$$

is a projection on $\mathcal{F}\left(G^{\mathrm{tr}}\right)$ that maps positive definite matrices to positive definite matrices and has the property

$$
\operatorname{trace}(A B)=\operatorname{trace}(A(B \pi))
$$

for all $A \in \mathcal{F}(G)$ and $B \in \mathbb{R}_{\text {sym }}^{d \times d}$.

Let $D:=\left\{q_{x}:=\left(x^{\text {tr }} x\right) \pi: x \in \mathbb{Z}^{d}\right\}$. Then for every $A \in \mathcal{F}(G)$ and $x \in \mathbb{Z}^{d}$

$$
\sigma\left(A, q_{x}\right)=\operatorname{trace}\left(A x^{\operatorname{tr}} x\right)=\operatorname{trace}\left(x A x^{\operatorname{tr}}\right)=x A x^{\operatorname{tr}} .
$$

So $\mu_{D}(A)$ for $A \in \mathcal{F}^{>0}(G)$ is the usual minimum $\mu(A)$ of $A$ as a positive definite quadratic form and 
the set $M_{D}(A)$ consists of the orthogonal projections onto the lines spanned by the shortest vectors. An algorithm for calculating the shortest vectors is described in [Pohst and Zassenhaus 1989].

Let $\left(A_{i}\right)_{i \in \mathbb{N}} \subset \mathcal{F}^{>0}(G)$ be a sequence converging to $A \in \partial \mathcal{F}^{>0}(G)$. Then $\operatorname{det}\left(A_{i}\right)$ converges to 0 . By Hermite there exists a constant $c$ depending only on the degree $d$ such that $\mu(B)^{d} \leq c \operatorname{det} B$ for every positive definite $B \in \mathbb{R}_{\mathrm{sym}}^{d \times d}$. Consequently $\mu\left(A_{i}\right)$ converges to 0 ; that is, $D$ is admissible.

A matrix $A$ is now called $G$-perfect (instead of $D$-perfect) if $M_{D}(A)$ contains a basis of $\mathcal{F}\left(G^{\text {tr }}\right)$. A.M. Bergé, J. Martinet and F. Sigrist gave a similar definition for $G$-perfect forms [Bergé and Martinet 1991; Bergé et al. 1992]. Instead of the projection $\pi$ they took the orthogonal projection (with respect to the trace) onto $\mathcal{F}(G)$. In general this projection does not map positive definite matrices to positive definite, but Theorem 3.6 does hold for both definitions.

Before stating the theorem the following definition is needed.

Definition 3.5. The Hermite function $\gamma:\left\{A \in \mathbb{R}_{\mathrm{sym}}^{d \times d} \mid\right.$ $A$ positive definite $\} \rightarrow \mathbb{R}$ is defined by

$$
\gamma(A):=\frac{\mu(A)}{(\operatorname{det} A)^{d}}
$$

for all positive definite matrices $A \in \mathbb{R}_{\mathrm{sym}}^{d \times d}$. Obviously $\gamma(\lambda A)=\gamma(A)$ for all $\lambda>0$. A matrix $A \in \mathcal{F}^{>0}(G)$ is called $G$-extreme if the restriction of $\gamma$ to $\mathcal{F}^{>0}(G)$ obtains a local maximum at $A$ in $\mathcal{F}^{>0}(G)$.

A positive definite matrix $A \in \mathbb{R}_{\mathrm{sym}}^{d \times d}$ is called $e u$ tactic if

$$
A^{-1}=\sum_{x \in M(A)} a_{x} x^{\operatorname{tr}} x
$$

with positive coefficients $a_{x}$, where $M(A)$ denotes the set of minimal vectors of $A$.

Theorem 3.6. (i) [Jaquet-Chiffelle 1995; Opgenorth 1996] There exist only finitely many G-perfect matrices up to the action of $N_{\mathbb{Z}}(G)$. (ii) [Bergé and Martinet 1991; Opgenorth 1996] $A$ matrix $A \in \mathcal{F}^{>0}(G)$ is $G$-extreme if and only if $A$ is $G$-perfect and eutactic.

This was already proved for the case $G=\left\{I_{d}\right\}$ by Voronoi [1908].

It remains to give a method for calculating $S_{A}$ the stabilizer in $N_{\mathbb{Z}}(G)$ for a Bravais group $G$ and a $G$-perfect matrix $A$. By Remark 3.2(iv) this means calculating generators for

$S_{A}=\left\{g \in \mathrm{GL}_{d}(\mathbb{Z}): g A g^{\mathrm{tr}}=A \wedge g \mathcal{F}(G) g^{\mathrm{tr}}=\mathcal{F}(G)\right\}$.

Let $\mathcal{R}$ denote the set of directions for $A$. Since $A$ is $G$-perfect $\mathcal{R}$ contains a basis $R_{1}, \ldots, R_{n}$ of $\mathcal{F}\left(G^{\mathrm{tr}}\right)$. As pointed out in Remark 2.4 the stabilizer of $A$ induces a permutation of $\mathcal{R}$. So

$$
\begin{aligned}
& S_{A}=\left\{g \in \mathrm{GL}_{d}(\mathbb{Z}): g A g^{\mathrm{tr}}=A\right. \text { and } \\
& \left.\qquad g R_{i} g^{\operatorname{tr}} \in \mathcal{R} \text { for } 1 \leq i \leq n\right\} .
\end{aligned}
$$

W. Plesken and B. Souvignier [1997] describe an algorithm to calculate generators of

$$
\text { Aut } A=\left\{g \in \mathrm{GL}_{d}(\mathbb{Z}): g A g^{\mathrm{tr}}=A\right\} .
$$

The basic idea of the algorithm is this: If $m$ is the maximal entry of the diagonal of $A$ and $M$ is the set of vectors $0 \neq x \in \mathbb{Z}^{d}$ with $x A x^{\text {tr }} \leq m$, the rows of a matrix $g \in$ Aut $A$ are elements of the finite set $M$. They construct the automorphisms row by row; that is, they construct a matrix $X \in Z^{r \times d}$ with rows from $M$ and try to extend this matrix to a matrix $\mathrm{Y}$ by adding a row from the vectors of $M$. For finding the next row the authors use several criteria. A generating set for $S_{A}$ can now be obtained by adding the criterion that for $1 \leq i \leq n$ the $(r+1) \times(r+1)$ matrix $Y R_{i} Y^{\text {tr }}$ has to occur as a left upper submatrix of one of the matrices in $\mathcal{R}$.

An alternative method to calculate the stabilizer of $A$ in $N_{\mathbb{Z}}(G)$ is to calculate the stabilizer $S_{A^{-1}}$ of $A^{-1}$ in $N_{\mathbb{Z}}\left(G^{\mathrm{tr}}\right)$, since $S_{A^{-1}}=S_{A}^{\mathrm{tr}}$. In this case one has to replace the set $\mathcal{R}$ by $M_{D}(A)$.

Plesken and Souvignier give in the same paper an analogous algorithm to decide whether or not there exists for two positive matrices $A$ and $B$ in $\mathbb{R}_{\text {sym }}^{d \times d}$ a matrix $X \in \mathrm{GL}_{d}(\mathbb{Z})$ with $X A X^{\mathrm{tr}}$. If such a matrix exists the algorithm constructs one. The same modification as above allows one to construct for $G$-perfect $A, B \in \mathcal{F}^{>0}(G)$ a matrix $X \in N_{\mathbb{Z}}(G)$ with $X A X^{\operatorname{tr}}=B$, if such a matrix exists. 
Example 3.7. Let $G \leq \mathrm{GL}_{n}(\mathbb{Z})$ be the group generated by

$$
g_{1}:=\left(\begin{array}{rrrrrrrr}
1 & 0 & -1 & -1 & 0 & 0 & 0 & 0 \\
-1 & 0 & 0 & 1 & 0 & 0 & 0 & 0 \\
1 & 1 & 0 & 0 & 0 & 0 & 0 & 0 \\
1 & -1 & -1 & -1 & 0 & 0 & 0 & 0 \\
0 & 0 & 0 & 0 & 1 & 0 & -1 & -1 \\
0 & 0 & 0 & 0 & -1 & 0 & 0 & 1 \\
0 & 0 & 0 & 0 & 1 & 1 & 0 & 0 \\
0 & 0 & 0 & 0 & 1 & -1 & -1 & -1
\end{array}\right), \quad g_{2}:=\left(\begin{array}{rrrrrrrr}
0 & 1 & 0 & 0 & 0 & 0 & 0 & 0 \\
-1 & -1 & 0 & 0 & 0 & 0 & 0 & 0 \\
0 & 1 & 0 & 1 & 0 & 0 & 0 & 0 \\
1 & 0 & -1 & -1 & 0 & 0 & 0 & 0 \\
0 & 0 & 0 & 0 & 0 & 1 & 0 & 0 \\
0 & 0 & 0 & 0 & -1 & -1 & 0 & 0 \\
0 & 0 & 0 & 0 & 0 & 1 & 0 & 1 \\
0 & 0 & 0 & 0 & 1 & 0 & -1 & -1
\end{array}\right), \quad g_{3}:=\left(\begin{array}{rrrrrrrr}
-1 & 0 & 0 & 0 & 0 & 0 & 0 & 0 \\
1 & 1 & 0 & 0 & 0 & 0 & 0 & 0 \\
-1 & 0 & 1 & 0 & 0 & 0 & 0 & 0 \\
0 & -1 & -1 & -1 & 0 & 0 & 0 & 0 \\
0 & 0 & 0 & 0 & 0 & 0 & -1 & 0 \\
0 & 0 & 0 & 0 & 0 & 1 & 1 & 1 \\
0 & 0 & 0 & 0 & -1 & 0 & 0 & 0 \\
0 & 0 & 0 & 0 & 1 & 0 & -1 & -1
\end{array}\right)
$$

$G$ is a finite unimodular group of order 48 isomorphic to $\mathrm{GL}_{2}(3)$. The space of invariant forms is of dimension 4. The algorithm leads to the following $G$-perfect forms

$$
P_{1}:=\left(\begin{array}{rrrrrrrr}
4 & -2 & 2 & 2 & 0 & -1 & 0 & 2 \\
-2 & 4 & -2 & -2 & 1 & 0 & -1 & 0 \\
2 & -2 & 4 & 0 & 0 & 1 & 0 & 1 \\
2 & -2 & 0 & 4 & -2 & 0 & -1 & 0 \\
0 & 1 & 0 & -2 & 4 & -2 & 2 & 2 \\
-1 & 0 & 1 & 0 & -2 & 4 & -2 & -2 \\
0 & -1 & 0 & -1 & 2 & -2 & 4 & 0 \\
2 & 0 & 1 & 0 & 2 & -2 & 0 & 4
\end{array}\right), \quad P_{2}:=\left(\begin{array}{rrrrrrrr}
2 & -1 & 1 & 1 & 0 & -1 & 0 & 2 \\
-1 & 2 & -1 & -1 & 1 & 0 & -1 & 0 \\
1 & -1 & 2 & 0 & 0 & 1 & 0 & 1 \\
1 & -1 & 0 & 2 & -2 & 0 & -1 & 0 \\
0 & 1 & 0 & -2 & 4 & -2 & 2 & 2 \\
-1 & 0 & 1 & 0 & -2 & 4 & -2 & -2 \\
0 & -1 & 0 & -1 & 2 & -2 & 4 & 0 \\
2 & 0 & 1 & 0 & 2 & -2 & 0 & 4
\end{array}\right)
$$

The form $P_{1}$ has minimum 4 and its determinant is $5^{4}$. It has four Voronoi neighbours, one of them is $P_{2}$, the three others are in the orbit $P_{1} N_{\mathbb{Z}}(G)$ and are permuted by the stabilizer $S_{P_{1}}$ of $P_{1}$ in $N_{\mathbb{Z}}(G)$. The stabilizer is generated by $g_{1}, g_{2}, g_{3}, s_{1}$ and $s_{2}$ where

$$
s_{1}:=\left(\begin{array}{rrrrrrrr}
0 & -1 & 0 & 0 & 0 & 0 & 0 & 0 \\
0 & 1 & 0 & 1 & 0 & 0 & 0 & 0 \\
-1 & -1 & 0 & 0 & 0 & 0 & 0 & 0 \\
1 & -1 & -1 & -1 & 0 & 0 & 0 & 0 \\
-1 & 1 & 1 & 1 & -1 & 0 & 1 & 1 \\
0 & -1 & -1 & -1 & 0 & 0 & -1 & 0 \\
0 & 1 & 1 & 0 & -1 & 0 & 1 & 0 \\
-1 & 0 & 1 & 1 & 0 & -1 & 0 & 0
\end{array}\right), \quad s_{2}:=\left(\begin{array}{rrrrrrrr}
0 & 0 & 0 & 0 & -1 & -1 & 0 & 0 \\
0 & 0 & 0 & 0 & 1 & 0 & 0 & -1 \\
0 & 0 & 0 & 0 & -1 & 0 & 1 & 1 \\
0 & 0 & 0 & 0 & 0 & -1 & -1 & 0 \\
-1 & -1 & 0 & 0 & 0 & 0 & 0 & 0 \\
1 & 0 & 0 & -1 & 0 & 0 & 0 & 0 \\
-1 & 0 & 1 & 1 & 0 & 0 & 0 & 0 \\
0 & -1 & -1 & 0 & 0 & 0 & 0 & 0
\end{array}\right)
$$

The form $P_{2}$ is isomorphic to the root lattice of $E_{8}$ and has minimum 2 and determinant 1 . $P_{2}$ has eight Voronoi neighbours (one of them $P_{1}$ ) which are all in one orbit of the stabilizer $S_{P_{2}}$ of $P_{2}$ in $N_{\mathbb{Z}}(G)$. The stabilizer is generated by $g_{1}, g_{2}, g_{3}, t_{1}, t_{2}$ and $t_{3}$, where

$$
t_{1}:=\left(\begin{array}{rrrrrrrr}
0 & 1 & 0 & 2 & 1 & 1 & 1 & 0 \\
0 & -2 & 0 & -1 & 1 & -1 & -2 & -1 \\
1 & 0 & -2 & 0 & 0 & 2 & 1 & 1 \\
-2 & 1 & 1 & 2 & 0 & 0 & 1 & 1 \\
3 & -1 & -1 & -2 & 1 & 0 & -1 & -2 \\
-1 & -1 & -2 & -1 & -1 & 1 & 0 & 2 \\
2 & 2 & 1 & -1 & -1 & 0 & 1 & -1 \\
2 & 0 & -1 & 1 & 2 & 1 & 0 & -1
\end{array}\right), \quad t_{2}:=\left(\begin{array}{rrrrrrrr}
-1 & 0 & -1 & 1 & 0 & 1 & 1 & 1 \\
2 & 0 & -1 & -2 & -1 & 0 & 0 & 0 \\
0 & -1 & -1 & 1 & 1 & 1 & 0 & 0 \\
-1 & 2 & 1 & 2 & 0 & 0 & 1 & 0 \\
1 & -3 & -2 & -3 & 0 & 0 & -1 & 0 \\
1 & 1 & 1 & 2 & 1 & 0 & 0 & -1 \\
-2 & -2 & 1 & -1 & 0 & -1 & -1 & 0 \\
0 & -2 & -3 & -1 & 0 & 1 & 0 & 1
\end{array}\right), \quad t_{3}:=\left(\begin{array}{rrrrrrrr}
0 & -1 & -1 & 0 & 0 & 0 & 0 & 0 \\
-1 & 1 & 1 & 1 & 0 & 0 & 0 & 0 \\
0 & -1 & -1 & -1 & 0 & 0 & 0 & 0 \\
1 & -1 & 0 & 0 & 0 & 0 & 0 \\
-1 & -1 & 0 & 1 & 1 & 1 & 0 & 0 \\
-1 & 1 & 1 & 0 & 0 & -1 & 0 & 0 \\
0 & -2 & -1 & -1 & 0 & 1 & 0 & 1 \\
0 & 0 & -1 & 1 & 0 & 1 & 1 & 0
\end{array}\right) .
$$

So we have the following residue graph $\Gamma_{D} / N_{\mathbb{Z}}(G)$ :

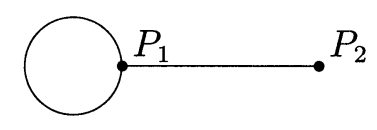


Let

$$
h:=\left(\begin{array}{rrrrrrrr}
0 & 0 & 0 & 0 & 0 & 1 & 0 & 0 \\
0 & 0 & 0 & 0 & 0 & 0 & 1 & 0 \\
0 & 0 & 0 & 0 & 0 & 1 & 0 & 1 \\
0 & 0 & 0 & 0 & -1 & 0 & 0 & 0 \\
0 & 0 & 0 & 1 & 1 & 0 & 0 & 0 \\
-1 & 0 & 0 & 0 & -1 & 0 & 0 & 1 \\
0 & -1 & 0 & 0 & 1 & -1 & -1 & -1 \\
1 & 0 & -1 & 0 & 1 & 1 & 0 & 0
\end{array}\right) .
$$

This matrix is in the normalizer $N_{\mathbb{Z}}(G)$ and maps $P_{1}$ to one of its neighbours. Therefore

$$
N_{\mathbb{Z}}(G)=\left\langle g_{1}, g_{2}, g_{3}, s_{1}, s_{2}, t_{1}, t_{2}, t_{3}, h\right\rangle .
$$

\section{ACKNOWLEDGEMENTS}

I thank Prof. W. Plesken for deep discussions. His experience and helpful hints have influenced many parts of this paper. My thanks also go to Gabriele Nebe, Rachel Camina and Anne Hilgers, who read a first draft of this paper and suggested corrections and improvements, and to all colleagues who have contributed to the development of CARAT on which the implementation of the described algorithms is based.

\section{REFERENCES}

[Bergé and Martinet 1991] A.-M. Bergé and J. Martinet, "Réseaux extrêmes pour un groupe d' automorphismes", pp. 41-66 in Journées arithmétiques (Luminy, 1989), edited by G. Lachaud, Astérisque 200, Soc. math. France, Paris, 1991.

[Bergé et al. 1992] A.-M. Bergé, J. Martinet, and F. Sigrist, "Une généralisation de l' algorithme de Voronoi", pp. 137-158 in Journées arithmétiques (Genève, 1991), edited by D. F. Coray and Y.-F. S. Petermann, Astérisque 209, Soc. math. France, Paris, 1992.

[Brown et al. 1973] H. Brown, J. Neubüser, and H. Zassenhaus, "On integral groups, III: Normalizers", Math. Comp. 27 (1973), 167-182.
[Dicks 1980] W. Dicks, Groups, trees and projective modules, Lecture Notes in Math. 790, Springer, Berlin, 1980.

[Jaquet-Chiffelle 1995] D.-O. Jaquet-Chiffelle, "Trois théorèmes de finitude pour les $G$-réseaux", J. Théor. Nombres Bordeaux 7:1 (1995), 165-176.

[Koecher 1957] M. Koecher, "Positivitätsbereiche im $\mathbb{R}^{n} "$, Amer. J. Math 79 (1957), 575-596.

[Koecher 1960] M. Koecher, "Beiträge zu einer Reduktionstheorie in Positivitätsbereichen, I", Math. Ann. 141 (1960), 384-432.

[Opgenorth 1996] J. Opgenorth, Normalisatoren und Bravaismannigfaltigkeiten endlicher unimodularer Gruppen, Verlag der Augustinus Buchhandlung, Aachen, 1996.

[Opgenorth et al. 1998] J. Opgenorth, W. Plesken, and T. Schulz, "Crystallographic algorithms and tables", Acta Cryst. Sect. A 54:5 (1998), 517-531. See http:// wwwb.math.rwth-aachen.de/carat/.

[Plesken and Schulz 2000] W. Plesken and T. Schulz, "Counting crystallographic groups in low dimensions", Experiment. Math. 9:3 (2000), 407-411.

[Plesken and Souvignier 1997] W. Plesken and B. Souvignier, "Computing isometries of lattices", $J$. Symbolic Comput. 24:3-4 (1997), 327-334.

[Pohst and Zassenhaus 1989] M. Pohst and H. Zassenhaus, Algorithmic algebraic number theory, Encyclopedia Math. Appl. 30, Cambridge University Press, Cambridge, 1989.

[Serre 1977] J.-P. Serre, Arbres, amalgames et $\mathrm{SL}_{2}$, Astérisque 46, Soc. math. France, Paris, 1977.

[Vinberg 1989] E. B. Vinberg, Linear representations of groups, Birkhäuser, Basel, 1989.

[Voronoi 1908] G. F. Voronoi, "Nouvelles applications des paramètres continus à la théorie des formes quadratiques, II: Recherches sur les parallélloèdres primitifs", J. Reine Angew. Math. 134 (1908), 198287.

Jürgen Opgenorth, RWTH Aachen, Lehrstuhl B für Mathematik, Templergraben 64, 52062 Aachen, Germany (juergen.opgenorth@sap.com)

Received July 9, 1999; accepted in revised form May 30, 2001 